Canad. Math. Bull. Vol. 21 (4), 1978

\title{
ON LEVINE'S DECOMPOSITION OF CONTINUITY
}

\author{
BY \\ DAVID ALON ROSE
}

\begin{abstract}
A strong version of Levine's decomposition of continuity leads to the result that a closed graph weakly continuous function into a rim-compact space is continuous. This result implies a closed graph theorem: every almost continuous closed graph function into a strongly locally compact space is continuous. An open problem of Shwu-Yeng T. Lin and Y.-F. Lin asks if every almost continuous closed graph function from a Baire space to a second countable space is necessarily continuous. This question is answered in the negative by an example.
\end{abstract}

1. Introduction. In $1961 \mathrm{~N}$. Levine [4] introduced weak continuity and weak* continuity for a function $f: X \rightarrow Y$ from a topological space $X$ into a topological space $Y$ and showed that $f$ is continuous if and only if $f$ is both weakly continuous and weak* continuous. The purpose of this note is to replace the weak* continuity by a weaker condition, local weak* continuity, and exercise the applicability of the resulting stronger decomposition theorem. As an application, a closed graph theorem of Paul E. Long and Earl E. McGehee Jr. [7] is slightly extended. An open problem of Shwu-Yeng T. Lin and Y.-F. Lin involving a similar closed graph theorem is answered in the negative by an example.

2. Definitions and preliminary results. Unless otherwise specified, no special properties will be assumed for the topological spaces $X$ and $Y$, or for a function $f: X \rightarrow Y$ from $X$ into $Y$. For a subset $A$ of a topological space $\mathrm{Cl}(A)$ and $\operatorname{Int}(A)$ denote the closure and interior of $A$ respectively.

Defintrion 1. [4]. A function $f: X \rightarrow Y$ is weakly continuous at the point $x$ in $X$ if and only if for each neighbourhood $V$ of $f(x)$ there is a neighbourhood $U$ of $x$ such that $f(U) \subseteq \mathrm{Cl}(V)$. A function $f: X \rightarrow Y$ is weakly continuous if and only if $f$ is weakly continuous at each point $x$ in $X$.

THEOREM 1. [4] A function $f: X \rightarrow Y$ is weakly continuous if and only if $f^{-1}(V) \subseteq \operatorname{Int}\left(f^{-1}(\mathrm{Cl}(V))\right)$ for each open subset $V$ of $Y$.

Received by the editors September 19, 1977 and, in revised form, December 16, 1977.

AMS (MOS) subject classifications (1970). Primary 54C10; Secondary 54C05, 54D45.

477 
THEOREM 2. A function $f: X \rightarrow Y$ is weakly continuous if and only if there is an open basis $B$ for the topology on $Y$ such that $f^{-1}(V) \subseteq \operatorname{int}\left(f^{-1}(\mathrm{Cl}(V))\right)$ for each $V$ in $B$.

THEOREM 3. [8] Every weakly continuous function $f: X \rightarrow Y$ into a Hausdorff space $Y$ has a closed graph, $G(f)$.

In $1966 \mathrm{~T}$. Husain [2] defined almost continuity and thus revived this condition which had been studied by H. Blumberg in 1922 [1] for real-valued functions on Euclidean space.

Definition 2. [2] A function $f: X \rightarrow Y$ is almost continuous if and only if for each $x$ in $X$ and for each neighbourhood $V$ of $f(x), \mathrm{Cl}\left(f^{-1}(V)\right)$ is a neighbourhood of $x$.

THEOREM 4. A function $f: X \rightarrow Y$ is almost continuous if and only if $f^{-1}(V) \subseteq$ $\operatorname{int}\left(\mathrm{Cl}\left(f^{-1}(V)\right)\right)$ for each open subset $V$ of $Y$.

Definition 3. [4] A function $f: X \rightarrow Y$ is weak* continuous if and only if $f^{-1}(\operatorname{Fr}(V))$ is closed in $X$ for each open subset $V$ of $Y$ where $\operatorname{Fr}(V)=$ $\mathrm{Cl}(V)-\operatorname{Int}(V)=\mathrm{Cl}(V)-V$ is the frontier (or boundary) of $V$.

Weak continuity and weak* continuity are independent conditions [4], weak continuity and almost continuity are independent conditions [10], and almost continuity and weak* continuity are independent conditions. Since every function into a discrete space is weak* continuous, Example 1 below shows that weak* continuity does not imply almost continuity. Example 3 of this paper demonstrates that almost continuity does not imply weak* continuity.

EXAMPLE 1. Let $X=R$ be the usual space of real numbers, $Y=R$ be the set of real numbers with the discrete topology, and let $f: X \rightarrow Y$ be the identity function.

3. Levine's decomposition of continuity. Levine's decomposition theorem [4] states that a function $f: X \rightarrow Y$ is continuous if and only if $f$ is weakly continuous and weak* continuous. The applicability of this result will be enhanced by replacing weak* ${ }^{*}$ continuity by local weak ${ }^{*}$ continuity as defined below.

Definition 4. A function $f: X \rightarrow Y$ is locally weak* continuous if and only if there is an open basis $B$ for the topology on $Y$ such that $f^{-1}(\operatorname{Fr}(V))$ is closed for each $V$ in $B$.

TheOREM 5. A function $f: X \rightarrow Y$ is continuous if and only if $f$ is weakly continuous and locally weak* continuous.

Proof. If $f$ is weakly continuous and locally weak* continuous and if $B$ is an open basis for the topology on $Y$ such that $f^{-1}(\mathrm{Fr}(V))=f^{-1}(\mathrm{Cl}(V))-f^{-1}(V)$ is 
closed for each $V$ in $B$, then

$$
f^{-1}(V)=\left(X-f^{-1}(\operatorname{Fr}(V))\right) \cap \operatorname{Int}\left(f^{-1}(\mathrm{Cl}(V))\right)
$$

is open for each $V$ in $B$ and $f$ is continuous. The converse is clear.

The following example shows that locally weak* continuous functions exist which are not weak* continuous.

EXAmple 2. Let $X=Y=R$ be the space of real numbers with the usual topology. Let $Q$ be the subset of rational numbers and let $Z$ be the subset of integers. Let $g: Q \rightarrow Z$ be a set equivalence (or bijection). Define $f: X \rightarrow Y$ by $f(x)=g(x)$ if $x$ is in $Q$ and $f(x)=x$ if $x$ is not in $Q$. Using the usual basis of open intervals $f$ is seen to be locally weak* continuous. But $f$ is not weak* continuous since $V=\bigcup\{(2 n, 2 n+1): n \in Z\}$ is open and $f^{-1}(\operatorname{Fr}(V))=f^{-1}(Z)=$ $Q$ is not closed.

4. Applications. A topological space $Y$ is rim-compact if and only if there is an open basis $B$ for the topology on $Y$ such that $\operatorname{Fr}(V)$ is compact for each $V$ in B. Paul E. Long and Larry L. Herrington [6] proved that an almost continuous $(S$ and $S$ ) closed graph function into a rim-compact space is necessarily continuous. This follows as a corollary to the following theorem since almost continuity ( $S$ and $S$ ) implies weak continuity [11].

THEOREM 6. Let $f: X \rightarrow Y$ be a weakly continuous function with a closed graph $G(f)$. If $Y$ is rim-compact then $f$ is continuous.

Proof. Let $B$ be an open basis for the topology on $Y$ such that $\operatorname{Fr}(V)$ is compact for each $V$ in $B$. Since $G(f)$ is closed, $f^{-1}(\operatorname{Fr}(V))$ is closed for each $V$ in $B$ by Problem $6 \mathrm{~A}$ of [3]. Thus $f$ is locally weak* continuous and hence continuous by Theorem 5 .

By Theorem 3 and Theorem 6, every weakly continuous function into a rim-compact Hausdorff space is continuous. But this is already known since weak continuity implies continuity when the range space is regular [4].

A topological space $Y$ is said to be strongly locally compact if each point of $Y$ has a closed compact neighbourhood. Every locally compact regular space is strongly locally compact and every strongly locally compact space is rimcompact. Since non-regular strongly locally compact spaces exist (Example 73 of [12]), a closed graph theorem of Paul E. Long and Earl E. McGehee Jr. [7] is slightly extended as follows.

THEOREM 7. If $f: X \rightarrow Y$ is an almost continuous function into a strongly locally compact space $Y$ and if $f$ has a closed graph, then $f$ is continuous.

Proof. Let $B$ be an open basis for the topology on $Y$ such that $\mathrm{Cl}(V)$ is compact for each $V$ in $B$. Since the graph of $f$ is closed, $f^{-1}(\mathrm{Cl}(V))$ is closed for 
each $V$ in $B$. Thus $\operatorname{Int}\left(\mathrm{Cl}\left(f^{-1}(V)\right)\right) \subseteq \operatorname{Int}\left(f^{-1}(\mathrm{Cl}(V))\right)$ for each $V$ in $B$ and by Theorem 4 and Theorem 2, $f$ is weakly continuous. Since $Y$ is rim-compact $f$ is continuous by Theorem 6 .

Corollary to Theorem 7. If $f: X \rightarrow R$ is an almost continuous closed graph real-valued function then $f$ is continuous.

5. Counterexample for an open problem. Shwu-Yeng T. Lin and Y.-F. Lin [5] recently posed the following open problem. If $f: X \rightarrow Y$ is an almost continuous closed graph function from a Baire space $X$ into a second countable space $Y$, then is $f$ necessarily continuous? This question is answered in the negative by the following example.

Example 3. Let $X=R$ be the usual space of real numbers and let $Q$ be the subset of rational numbers. Let $Y=R$ be the space of real numbers topologized with the smallest extension of the usual topology for which $Q$ is open in $Y$. Then $X$ is a Baire space and if $\left\{V_{n}\right\}$ is a countable open basis for the usual topology on $X$ then $\left\{V_{n}\right\} \cup\left\{V_{n} \cap Q\right\}$ is a countable open basis for the extension topology on $Y$ showing that $Y$ is a second countable space. Clearly $Y$ is a Hausdorff (and Urysohn) space. Let $f: X \rightarrow Y$ be the identity function. By Theorem $2, f$ is weakly continuous since $f^{-1}(V) \subseteq \operatorname{Int}\left(f^{-1}(\mathrm{Cl}(V))\right)$ for each $V=(a, b)$ and for each $V=(a, b) \cap Q$ where $a<b$. Further, $f$ is clearly open but $f$ is discontinuous since $f^{-1}(Q)=Q$ is not open in $X$. It is noted in [10] that every open weakly continuous function is almost continuous. Thus $f$ is almost continuous and by Theorem 3 the graph of $f$ is closed. Further, by Theorem $5 f$ is not locally weak* continuous.

\section{REFERENCES}

1. H. Blumberg, New properties of all real functions, Trans. Amer. Math. Soc. 24 (1922), $113-128$.

2. T. Husain, Almost continuous mappings, Prace Mat. 10 (1966), 1-7.

3. John L. Kelley, General Topology, D. Van Nostrand Co., Inc., Princeton, New Jersey, 1955.

4. Norman Levine, A decomposition of continuity in topological spaces, Amer. Math. Monthly 68 (1961), 44-46.

5. Shwu-Yeng T. Lin and Y.-F. Lin, On almost continuous mappings and Baire spaces, Canadian Mathematical Bulletin, Vol. 21, No. 2, pages

6. Paul E. Long and Larry L. Herrington, Properties of almost-continuous functions, Boll. Un. Mat. Ital. 10 (1974), 336-342.

7. Paul E. Long and Earl E. McGehee Jr., Properties of almost continuous functions, Proc. Amer. Math. Soc. 24 (1970), 175-180.

8. Takashi Noiri, Between continuity and weak continuity, (Italian Summary) Boll. Un. Mat. Ital. 9 (1974), 647-654.

9. Takashi Noiri, On weakly continuous mappings, Proc. Amer. Math. Soc. 46 (1974), 120-124.

10. David Alon Rose, Weak continuity and almost continuity, Pacific Journal of Mathematics, to appear.

11. M. K. Singal and Asha Rani Singal, Almost-continuous mappings, Yokohama Math. Journal 16 (1968), 63-73. 
12. Lynn A. Steen and J. Arthur Seebach Jr., Counterexamples in Topology, Holt, Rinehart and Winston, Inc., New York, 1970.

Division of SCIENCE AND Mathematics

UNIVERSITY OF TAMPA

TAMPA, FLORIDA 33606 